\title{
Association between GeneXpert Diagnosis and Same-Day Initiation of Tuberculosis Treatment in Rural Eastern Uganda
}

\author{
Jonathan Izudi, ${ }^{*}$ Imelda K. Tamwesigire, and Francis Bajunirwe \\ Department of Community Health, Faculty of Medicine, Mbarara University of Science and Technology, Mbarara, Uganda
}

\begin{abstract}
Diagnosis of tuberculosis with GeneXpert and same-day initiation of tuberculosis treatment (SITT) has important public health and clinical benefits. GeneXpert allows for rapid diagnosis, hence presenting an opportunity for SITT. We determined the association between GeneXpert diagnosis and SITT, and the effect of SITT on treatment success rate among adult persons with bacteriologically confirmed pulmonary tuberculosis (BC-PTB) in rural eastern Uganda. We conducted a retrospective cohort study using routinely collected data at 10 health facilities, used modified Poisson regression analysis to determine the association between GeneXpert and SITT, and expressed the results as risk ratio (RR). We used propensity score-matched analysis to match SITT participants to delayed initiation of treatment (DIT) participants and then performed logistic regression analysis to determine the independent effect of SITT on treatment success rate, expressed as odds ratio (OR). Of 1,045 participants, 764 (73.1\%) had SITT. The use of GeneXpert for diagnosis of tuberculosis was associated with higher likelihood of SITT (adjusted RR [aRR], 1.28; 95\% CI: 1.19-1.37) and for persons with new BC-PTB diagnosis (aRR, 1.16; 95\% Cl: 1.02-1.32). We successfully matched 530 participants (265 in SITT and 265 in DIT) through 1:1 nearest neighbor caliper matching. Before propensity score-matched analysis, SITT was not significantly associated with treatment success rate (adjusted OR, 0.97; 95\% Cl: 0.70-1.33). After propensity scorematched analysis, SITT remained nonsignificantly associated with treatment success rate (OR, 0.96; 95\% Cl: 0.67-1.40). GeneXpert thus increases the likelihood of SITT, but SITT has no significant effect on treatment success rate.
\end{abstract}

\section{INTRODUCTION}

The 2019 Global Tuberculosis Report indicates that 10 million people developed tuberculosis disease in 2018, of which 1.5 million died. ${ }^{1}$ Tuberculosis is an airborne disease, and persons with untreated tuberculosis disease unknowingly infect between 10 and 15 other persons annually on average. ${ }^{2}$ The risk of infection is reduced when tuberculosis disease is diagnosed and treated early. ${ }^{3}$ Early treatment of tuberculosis confers important clinical and broader public health benefits of minimizing complications ${ }^{4}$ and reducing transmission. However, several health system and individual-level factors continue to curtail and delay the implementation of early diagnosis of tuberculosis and treatment initiation. For example, along the tuberculosis treatment pathway, there are patient, healthcare provider, diagnostic, and treatment delays. ${ }^{3,5}$ These delays accelerate tuberculosis disease progression and consequently results in more severe symptoms of tuberculosis, ${ }^{6}$ thus propagating its transmission.

To address treatment delays, the time interval between diagnosis of tuberculosis disease and treatment initiation, the World Health Organization (WHO) has since 2011 recommended same-day diagnosis of tuberculosis, and this approach involves same-day collection of two consecutive sputum smear specimens for microscopy at patient consultation. ${ }^{7}$ It is anticipated that same-day diagnosis would enable healthcare providers to initiate tuberculosis treatment on the same day as consultation. ${ }^{7}$ In other words, persons with tuberculosis receive same-day initiation of tuberculosis treatment (SITT) as diagnosis. To achieve SITT, rapid diagnostic tools are needed to provide quick turnaround time and reduce diagnostic delays, the time lag between sample collection and diagnosis of tuberculosis.

\footnotetext{
*Address correspondence to Jonathan Izudi, Department of Community Health, Faculty of Medicine, Mbarara University of Science and Technology, Kabale Road, P.O. Box, 1410, Mbarara, Uganda. E-mail: jonahzd@gmail.com
}

Clinicians require a confirmed diagnosis of tuberculosis before treatment decision is made. Studies have shown that same-day approaches to diagnosis of tuberculosis are feasible and are as accurate as conventional approaches such as sputum smear microscopy. ${ }^{8,9}$ Xpert ${ }^{\circledR}$ Mycobacterium tuberculosis and rifampicin (Xpert ${ }^{\circledR}$ MTB/RIF, Cepheid, Sunnyvale, $\mathrm{CA})$ resistance commonly known as GeneXpert is one of the simple, rapid, and accurate diagnostic tools for detecting MTB in sputum specimens. ${ }^{10}$ Accordingly, GeneXpert has been extensively scaled up in most resource-limited settings to provide a rapid turnaround of results and is hence widely expected to promote SITT. However, data are scarce on the magnitude of SITT and whether GeneXpert diagnosis at health facilities increases the likelihood of SITT among persons with tuberculosis. In addition, although SITT is expected to improve clinical outcomes, no study has evaluated its effect on treatment success rate.

In this study, our primary objective was to determine the association between GeneXpert diagnosis and SITT among adult persons with bacteriologically confirmed pulmonary tuberculosis (BC-PTB) in rural eastern Uganda. The secondary objective was to evaluate the effect of SITT on treatment success rate among adult persons with BC-PTB in rural eastern Uganda.

\section{METHODS AND MATERIALS}

Study setting. This study was conducted at 10 health facilities spread across four districts, namely, Soroti, Serere, Kumi, and Ngora that comprise the Teso region in rural eastern Uganda. Although some of the health facilities are located in an urban setting, they all serve a predominantly rural population. Over $80 \%$ of Uganda's population is rural, and in eastern Uganda, over $90 \%$ of the population is rural and the region is considered a rural setting.

We obtained the district treatment success rate data from the most recent Annual Health Sector Performance Report of Uganda, and using the WHO optimum treatment success rate 
target of at least $90 \%$, we categorized districts with a treatment success rate $\geq 90 \%$ as high and those $<90 \%$ as low. ${ }^{11,12}$ The 10 health facilities were purposively selected because they had high patient loads of at least 100 persons with tuberculosis per year based on routine data. For Soroti district, the health facilities included were Soroti Regional Referral Hospital, Princess Diana Memorial Health Center (HC) IV, and Tiriri HC IV, and for Serere district, Serere and Apapai HC IVs were included. In Ngora district, Ngora HC IV and Ngora Hospital were the study sites, and Kumi HC IV, Kumi Hospital, and Atutur Hospital were the health facilities included in Kumi district. A HC IV is a county-level health facility.

The Uganda Ministry of Health launched GeneXpert for the diagnosis of pulmonary tuberculosis and rifampicin-resistant and multidrug-resistant tuberculosis at selected health facilities in Uganda in July $2017 .^{13}$ In our study area, four sites, namely, Soroti Regional Referral Hospital, Serere HC IV, Atutur Hospital, and Ngora HC IV were selected to provide GeneXpert. This selection was guided by geographical reach to enable easy access, particularly for peripheral health facilities. Six study sites were not provided with GeneXpert, and the primary mode of diagnosis of tuberculosis is sputum smear microscopy, but GeneXpert is available off-site. For these sites, sputum samples for GeneXpert are transported through a hub system, ${ }^{13}$ a system for transportation of samples and return of results. Apart from Soroti Regional Referral Hospital, all the other sites are county-level health facilities and offer the same range of services. Microscopy is still available as a diagnostic tool at all the sites.

Each hub system has two hub riders mandated to collect, transport, and deliver samples from non-GeneXpert to GeneXpert sites on specific days of the week, and to return the results thereafter. When samples need quick transportation, a backup hub rider is available. Each district has a district hub coordinator to plan, organize, coordinate, and manage the hub system. At non-GeneXpert sites, GeneXpert results are used to rule out rifampicin-resistant or multidrug-resistant tuberculosis, and to confirm initial diagnosis made by sputum smear microscopy.

Study design and eligibility. We used routine records at the tuberculosis clinics to construct a retrospective cohort study for adult persons ( $\geq 18$ years) with BC-PTB diagnosed and treated between January 2015 and June 2018. In the clinic registers, clinicians enter records of patients starting from the date of treatment initiation to treatment completion, approximately 6 months for persons with new BC-PTB diagnosis and 8 months for previously treated persons with BC-PTB. The participants were either persons with new BCPTB diagnosis or previously treated persons with BC-PTB. For participants who were transferred in, this should not have happened more than 2 months into their tuberculosis treatment at the referring health facilities. We excluded participants with no data on the date of diagnosis of tuberculosis and treatment initiation.

Measurements. We extracted data on patients' clinical, sociodemographic, and health system-related factors, namely the level of health facility, and treatment outcomes specifically cure and treatment completion for the primary and secondary objectives. The primary outcome variable was SITT measured as a dichotomous variable (yes or no). We considered the dates of sputum smear collection, diagnosis of tuberculosis, and antituberculosis treatment initiation from the registers to determine who had SITT. Participants with same dates of sputum smear collection, diagnosis, and treatment initiation were considered to have had SITT, and all the others were regarded as delayed initiation of treatment (DIT).

We defined a person with BC-PTB as one with a biological specimen that is positive for MTB on smear microscopy, culture, or GeneXpert before treatment initiation. The independent variables we considered for SITT included patient characteristics, namely, age, gender, type of person with BCPTB (new diagnosis or previously treated), mode of diagnosis of tuberculosis (sputum smear microscopy or GeneXpert), HIV infected (no or yes), and type of directly observed therapy short course (facility or community) recorded in the tuberculosis register. The health service-related factors were the district treatment success rate, level of the health facility $(\mathrm{HC}$ IV also known as county-level $\mathrm{HC}$, district hospital, and referral hospital), location of the health facility (peri-urban or rural), and health facility ownership (public or private not-for-profit). We also categorized health facilities located within a 5-km radius to the main trading town as peri-urban and those beyond 5-km radius as rural.

For the secondary objective, the exposure variable was treatment initiation, namely, SITT as the intervention group and DIT as the comparison group. The outcome was treatment success rate derived from treatment outcomes for tuberculosis, measured in accordance with the WHO criteria detailed as follows. ${ }^{14}$

1. Cured: A person with BC-PTB who was smear or culture negative in the last month of treatment and on at least one previous occasion, either at the 5th month or at the end of intensive phase of treatment.

2. Lost to follow-up: A person with BC-PTB who did not start treatment or whose treatment was interrupted for more than two consecutive months.

3. Treatment completed: A person with BC-PTB who completed treatment without evidence of cure.

4. Treatment failed: A person with BC-PTB whose sputum smear or culture result was positive in the 5th month of treatment or later during treatment.

5. Dead: A person with BC-PTB who died for any reason before starting treatment or during treatment.

6. Treatment not evaluated: The number of persons with $\mathrm{BC}-$ PTB whose treatment outcome could not be evaluated either because they were transferred to other health facilities or because the treatment outcome was missing in the tuberculosis unit register.

We categorized participants with treatment outcomes of cure or treatment completed as treatment success and those with outcomes of treatment failed, lost to follow-up, and dead as unsuccessful treatment. We computed treatment success rate as the proportion of persons with BC-PTB with an outcome of treatment success.

We used propensity score-matched analysis to create two groups of persons with BC-PTB, SITT, and DIT categories, with closely balanced covariates to remove selection bias and confounding. The matching covariates were variables hypothesized as associated with treatment initiation (the exposure) and treatment success rate (the outcome), as recommended in propensity score-matched analysis. ${ }^{15,16}$ We identified 13 matching covariates, namely, the tuberculosis regimen ( 2 months of rifampicin 
$[R]$, isoniazid $[H]$, pyrazinamide [Z], ethambutol/4 months of $\mathrm{RH}$ [2RHZE/4RH]; 2RHZE/6 months of HE; or 2RHZE, streptomycin/ 1 month of RHZE/5 months of RHE), year of tuberculosis treatment (2015-2018), patient residence or whether the patient lived in the same subcounty as the health facility (yes or no), and the 10 other independent variables (age, gender, type of persons with BC-PTB, mode of diagnosis of tuberculosis, HIV infection, type of directly observed therapy short course, and health facility attributes, namely, the district, level, location, and ownership type that were included in the primary analysis).

Data analysis. Primary analysis. In the univariate analysis, we computed descriptive statistics of mean and standard deviation (SD) for numerical data and percentages for categorical data. In the bivariate analysis, we used the chi-squared test to compare differences in proportions for categorical variables such as gender between participants with SITT and those with DIT for large cell counts, and the Fisher's exact test was used for smaller cell counts. The Student's $t$-test was used to compare mean differences for numerical variables, for example, age. In the multivariate analysis, we used the modified Poisson regression analysis with robust standard errors to compute RRs because the primary outcome was frequent (more than 10\%), and odds ratios (ORs) would overestimate the degree of association. ${ }^{17}$ We reported each $\mathrm{RR}$ with subsequent $95 \% \mathrm{Cl}$.

Secondary analysis. We used propensity score-matched analysis to achieve balance or comparability in observed covariates between participants in the SITT and DIT groups, thereby approximating a randomized controlled trial. ${ }^{18,19}$ First, we compared the distribution of participants' baseline characteristics between the SITT and DIT groups before and after propensity score-matched analysis using standardized mean differences (SMDs), and SMDs less than 0.25 were considered indicative of good balance. ${ }^{20}$

We then generated propensity scores by regressing the 13 matching covariates on the intervention (SITT) in a logit model and then balanced the generated propensity scores across the SITT and DIT groups. We explored and confirmed balance in propensity scores across the SITT and DIT groups with a jitter plot and histogram and considered distributional similarity in propensity scores as indicative of good balance.

After balancing the propensity scores, we matched participants in the SITT group to those in the DIT group on similar propensity scores using nearest neighbor $1: 1$ caliper matching, and thereafter confirmed covariate balance using SMD. After successful covariate balance, we performed both unadjusted and adjusted binary logistic regression analyses to establish the unbiased effect of SITT on treatment success rate and reported the results as ORs with $95 \% \mathrm{Cl}$.

In addition, we performed sensitivity analysis to assess how robust our results are to hidden bias, biases not identified in the study or removed by propensity score-matched analysis, using Rosenbaum Wilcoxon's signed rank test. We interpreted the resulting value of gamma as the odds of treatment assignment hidden bias. ${ }^{20} \mathrm{~A}$ gamma value close to 1 implied that the result was not free from hidden bias, whereas distant gamma values from 1 indicated the absence of hidden bias or removal of bias by propensity scorematched analysis.

All the analyses were performed in $\mathrm{R}$ programming language and statistical software version 3.5.2 (R Foundation for Statistical Computing Platform, Vienna, Austria) ${ }^{21}$ at $5 \%$ level of significance. For propensity score-matched analysis, we used the "Matchlt" package in $\mathrm{R}^{22,23}$ We adhered to the elements of Strengthening of the Reporting of Observational Studies in Epidemiology guideline ${ }^{24,25}$ in reporting the primary outcome analysis, and propensity score-matched analysis guideline for the secondary outcome. ${ }^{26}$

Ethical issues. This study was approved by Mbarara University of Science and Technology Research Ethics Committee (reference number 03/11-18) and the Uganda National Council of Science and Technology (reference number HS 2531).

We requested for a waiver of consent from the Research Ethics Committee at Mbarara University of Science and Technology to access patient records, given the large number of records and the difficulty in reaching all the adult persons with BC-PTB to obtain individual consent. We received the approval from the ethics committee, and permission was granted to access patient records to extract secondary data. Personal identifiers such as names and hospital identification numbers were not abstracted.

\section{RESULTS}

Characteristics of participants. We extracted records of 1,045 participants, and their mean age was 38.7 years $(S D=$ 15.4). Of these, only 764 (73.1\%) had received SITT. Participants who had SITT were slightly younger than those who had DIT: $38.2 \pm 15.3$ versus $40.2 \pm 5.6$, respectively, but the difference was not statistically significant $(P=0.068)$. Participants in the SITT and DIT groups were different with respect to the following baseline characteristics: the district treatment success rate, level of health facility, type of person with BCPTB, and mode of diagnosis of tuberculosis. Table 1 presents the distribution of participants' characteristics between SITT and DIT groups.

Factors associated with SITT. In unadjusted analysis (Table 2), SITT was more likely when the participant was from a district with a high treatment success rate (RR, 1.16; 95\% Cl: 1.08-1.25), had new BC-PTB diagnosis (RR, 1.16; $95 \% \mathrm{Cl}$ : 1.01-1.32) and when the tuberculosis was diagnosed by GeneXpert (RR, 1.30; 95\% Cl: 1.22-1.39). Participants at sites with GeneXpert were not any more likely to have SITT than those without GeneXpert (RR, 1.04; 95\% Cl: 0.96-1.13). Conversely, participants who had received treatment at higher level health facilities, namely, district (RR, 0.85; $95 \% \mathrm{Cl}$ : $0.77-0.95)$ and referral hospitals (RR, 0.89; $95 \% \mathrm{Cl}: 95 \% \mathrm{Cl}$ : 0.82-0.96) were less likely to have SITT than those at HC IV, which is relatively a lower level health facility.

In adjusted analysis, patients diagnosed by GeneXpert were $28 \%$ more likely to receive SITT than those diagnosed by sputum smear microscopy (adjusted RR [aRR], 1.28; 95\% Cl: 1.19-1.37). Participants with new BC-PTB diagnosis were $16 \%$ more likely to receive SITT than those with a history of previous tuberculosis treatment $(\mathrm{aRR}, 1.16 ; 95 \% \mathrm{Cl}$ : 1.02-1.25).

We examined whether prior treatment for tuberculosis might modify the effect of GeneXpert on SITT. We found no statistically significant interaction (test of homogeneity, $P=$ 0.569), suggesting GeneXpert influenced SITT in the same way among persons with new and those with previously treated BC-PTB. We also examined for potential effect modification by having GeneXpert on-site versus off-site. We 
TABLE 1

Baseline characteristics of participants with BC-PTB in rural eastern Uganda

\begin{tabular}{|c|c|c|c|c|c|}
\hline \multirow[b]{2}{*}{ Variable } & \multirow[b]{2}{*}{ Level } & \multicolumn{2}{|c|}{ Treatment initiation } & \multirow[b]{2}{*}{$\begin{array}{c}\text { Total, } \\
n=1,045(\%)\end{array}$} & \multirow[b]{2}{*}{$P$-value } \\
\hline & & $\begin{array}{l}\text { Delayed initiation of } \\
\text { treatment, } n=281(\%)\end{array}$ & $\begin{array}{l}\text { Same-day initiation of } \\
\text { treatment, } n=764(\%)\end{array}$ & & \\
\hline \multirow[t]{2}{*}{ District treatment success rate } & Low $(<90 \%)$ & $226(80.4)$ & $528(69.1)$ & $754(72.1)$ & \multirow[t]{2}{*}{$<0.001$} \\
\hline & High ( $\geq 90 \%)$ & 55 (19.6) & 236 (30.9) & 291 (27.9) & \\
\hline \multirow[t]{3}{*}{ Level of health facility } & Health center IV & $86(30.6)$ & $324(42.4)$ & $410(39.2)$ & \multirow[t]{3}{*}{0.002} \\
\hline & General hospital & $73(26.0)$ & 152 (19.9) & $225(21.5)$ & \\
\hline & Regional hospital & $122(43.4)$ & $288(37.7)$ & $410(39.2)$ & \\
\hline \multirow[t]{3}{*}{ Age-group (years) } & $15-34$ & $121(43.1)$ & 354 (46.3) & $475(45.4)$ & \multirow[t]{3}{*}{0.631} \\
\hline & $35-50$ & 99 (35.2) & 257 (33.6) & $356(34.1)$ & \\
\hline & $>50$ & $61(21.7)$ & $153(20.0)$ & $214(20.5)$ & \\
\hline Age, years (numerical) & Mean (SD) & $40.2(15.59)$ & $38.2(15.3)$ & 38.7 (15.4) & 0.068 \\
\hline \multirow[t]{2}{*}{ Gender } & Male & 189 (67.3) & 496 (64.9) & $685(65.6)$ & \multirow[t]{2}{*}{0.527} \\
\hline & Female & $92(32.7)$ & $268(35.1)$ & $360(34.4)$ & \\
\hline \multirow[t]{2}{*}{ Type of persons with BC-PTB } & Previously treated & $46(16.4)$ & 83 (10.9) & $29(12.3)$ & \multirow[t]{2}{*}{0.022} \\
\hline & New & 235 (83.6) & 681 (89.1) & $916(87.7)$ & \\
\hline \multirow[t]{2}{*}{ Mode of diagnosis of tuberculosis } & Microscopy & 247 (87.9) & $517(67.1)$ & $764(73.1)$ & \multirow[t]{2}{*}{$<0.001$} \\
\hline & GeneXpert & $34(12.1)$ & 247 (32.3) & 281 (26.9) & \\
\hline \multirow{2}{*}{$\begin{array}{l}\text { Type of directly observed therapy short } \\
\text { course }\end{array}$} & Health facility & $17(6.0)$ & $30(3.9)$ & $47(4.5)$ & \multirow[t]{2}{*}{0.194} \\
\hline & Community & $264(94.0)$ & $734(96.1)$ & $998(95.5)$ & \\
\hline \multirow[t]{2}{*}{ Treatment supporter } & No & $33(11.7)$ & $94(12.3)$ & $127(12.2)$ & \multirow[t]{2}{*}{0.890} \\
\hline & Yes & 248 (88.3) & $670(87.7)$ & $918(87.8)$ & \\
\hline \multirow[t]{2}{*}{ HIV infected } & No & 199 (72.1) & $522(68.9)$ & $721(69.7)$ & \multirow[t]{2}{*}{0.355} \\
\hline & Yes & $77(27.9)$ & $236(31.1)$ & $313(30.3)$ & \\
\hline
\end{tabular}

BC-PTB = bacteriologically confirmed pulmonary tuberculosis; SITT = same-day initiation of treatment.

found no significant difference in the influence of GeneXpert on SITT in the two settings (test of homogeneity, $P=0.66$ ). We examined the time between sputum collection and treatment initiation in the DIT category. The median time to treatment in this group was 2 days with an interquartile range of $1-10$ days.

Participant baseline characteristics before and after propensity score-matched analysis. Table 3 summarizes the baseline characteristics of participants included in propensity score-matched analysis stratified by SITT and DIT groups. Before propensity score-matched analysis, there were 764 (73.1\%) participants in the SITT and $281(26.7 \%)$ in the DIT groups, differing significantly (SMD $\geq 0.25$ ) with respect to the district where treatment was received, health facility ownership, level of health facility, year of tuberculosis treatment, mean age, type of persons with BC-PTB, mode of diagnosis of tuberculosis, and antituberculosis regimen.
However, after propensity score-matched analysis, there were 265 (50.0\%) participants in either group, similar to all the baseline characteristics (SMD < 0.25).

Matching approach and balance diagnostics. Figure 1 presents a jitter plot showing the distribution of propensity scores represented by a circle before and after propensity score-matched analysis. In the unmatched group, we noticed clustering of observations towards higher propensity scores, and this changed to a uniform distribution when matching was carried out. We further examined and confirmed the graphical distribution of propensity scores using a histogram which showed uniform distribution of propensity scores after matching. The matching was performed using the nearest neighbor 1:1 caliper method because it balanced all the covariates between the two groups (SMD < 0.25). Our caliper was 0.03 , calculated as $20 \%$ of the SD of propensity scores. ${ }^{27}$ The nearest neighbor caliper matching equally showed the

TABLE 2

Factors associated with SITT in rural eastern Uganda

\begin{tabular}{|c|c|c|c|c|c|c|c|}
\hline \multirow[b]{2}{*}{ Variable } & \multirow[b]{2}{*}{ Level } & \multicolumn{2}{|c|}{ Treatment initiation } & \multicolumn{2}{|c|}{ Crude analysis } & \multicolumn{2}{|c|}{ Adjusted analysis } \\
\hline & & $\begin{array}{l}\text { Delayed initiation of } \\
\text { treatment, } n=281(\%)\end{array}$ & $\begin{array}{c}\text { SITT, } \\
n=764(\%)\end{array}$ & $\mathrm{RR}$ & $95 \% \mathrm{Cl}$ & $\begin{array}{l}\text { Adjusted } \\
\text { RR }\end{array}$ & $95 \% \mathrm{Cl}$ \\
\hline \multirow[t]{2}{*}{ District treatment success rate } & Low (<90\%) & $226(80.4)$ & $528(69.1)$ & 1 & - & 1 & - \\
\hline & High ( $\geq 90 \%)$ & 55 (19.6) & $236(30.9)$ & $1.16^{\star \star}$ & $(1.08-1.25)$ & 1.06 & $(0.96-1.17)$ \\
\hline \multirow[t]{3}{*}{ Level of health facility } & Health center IV & $86(30.6)$ & $324(42.4)$ & 1 & - & 1 & - \\
\hline & General hospital & $73(26.0)$ & 152 (19.9) & $0.85^{\star \star}$ & $(0.77-0.95)$ & 0.91 & $(0.81-1.02)$ \\
\hline & Regional hospital & $122(43.4)$ & $288(37.7)$ & $0.89^{\star \star}$ & $(0.82-0.96)$ & 0.96 & $(0.86-1.07)$ \\
\hline \multirow{2}{*}{$\begin{array}{l}\text { Type of persons with bacteriologically } \\
\text { confirmed pulmonary tuberculosis }\end{array}$} & Previously treated & $46(16.4)$ & 83 (10.9) & 1 & - & 1 & - \\
\hline & New diagnosis & 235 (83.6) & $681(89.1)$ & $1.16^{\star}$ & $(1.01-1.32)$ & $1.16^{*}$ & $(1.02-1.32)$ \\
\hline \multirow[t]{2}{*}{ Mode of diagnosis of tuberculosis } & Microscopy & $247(87.9)$ & $517(67.1)$ & 1 & - & 1 & - \\
\hline & GeneXpert & $34(12.1)$ & 247 (32.3) & $1.30^{\star \star \star}$ & $(1.22-1.39)$ & $1.28^{\star \star \star}$ & $(1.19-1.37)$ \\
\hline \multirow[t]{2}{*}{ GeneXpert site } & No & $94(33.5)$ & $232(30.4)$ & 1 & - & - & - \\
\hline & Yes & $187(66.5)$ & $532(69.6)$ & 1.04 & $(0.96-1.13)$ & - & - \\
\hline
\end{tabular}

${ }^{*} P<0.05$.

${ }^{\star \star} P<0.01$.

${ }^{* \star \star} P<0.001$. 
TABLE 3

Participants' baseline characteristics before and after propensity score-matched analysis

\begin{tabular}{|c|c|c|c|c|c|c|c|}
\hline \multirow[b]{2}{*}{ Characteristic } & \multirow[b]{2}{*}{ Level } & \multicolumn{3}{|c|}{ Unmatched cohort data } & \multicolumn{3}{|c|}{ Matched cohort data } \\
\hline & & DIT & SITT & SMD & DIT & SITT & SMD \\
\hline \multirow{5}{*}{$\begin{array}{l}\text { Number of participants } \\
\text { District of health facility location }\end{array}$} & & 281 & 764 & & 265 & 265 & \\
\hline & Soroti & $140(49.8)$ & $352(46.1)$ & 0.329 & $134(50.6)$ & $140(52.8)$ & 0.113 \\
\hline & Kumi & $86(30.6)$ & $176(23.0)$ & & $78(29.4)$ & $71(26.8)$ & \\
\hline & Ngora & 35 (12.5) & 107 (14.0) & & 33 (12.5) & 39 (14.7) & \\
\hline & Serere & $20(7.1)$ & 129 (16.9) & & $20(7.5)$ & $15(5.7)$ & \\
\hline \multirow[t]{2}{*}{ Type of health facility ownership } & Public & $236(84.0)$ & 677 (88.6) & 0.135 & $222(83.8)$ & $221(83.4)$ & 0.010 \\
\hline & Private not-for-profit & 45 (16.0) & $87(11.4)$ & & $43(16.2)$ & $44(16.6)$ & \\
\hline \multirow[t]{3}{*}{ Level of health facility } & Health center IV & 86 (30.6) & $324(42.4)$ & 0.252 & 81 (30.6) & $85(32.1)$ & 0.041 \\
\hline & District hospital & $73(26.0)$ & 152 (19.9) & & $68(25.7)$ & 64 (24.2) & \\
\hline & Referral hospital & $122(43.4)$ & 288 (37.7) & & $116(43.8)$ & $116(43.8)$ & \\
\hline \multirow[t]{2}{*}{ Location of health facility } & Rural & $79(28.1)$ & $222(29.1)$ & 0.021 & $76(28.7)$ & 79 (29.8) & 0.025 \\
\hline & Peri-urban & 202 (71.9) & 542 (70.9) & & 189 (71.3) & 186 (70.2) & \\
\hline \multirow[t]{4}{*}{ Year of tuberculosis treatment } & 2015 & $121(43.1)$ & 265 (34.7) & 0.238 & $114(43.0)$ & 110 (41.5) & 0.110 \\
\hline & 2016 & $69(24.6)$ & $166(21.7)$ & & $63(23.8)$ & $55(20.8)$ & \\
\hline & 2017 & $47(16.7)$ & $182(23.8)$ & & $47(17.7)$ & 50 (18.9) & \\
\hline & 2018 & 44 (15.7) & $151(19.8)$ & & 41 (15.5) & $50(18.9)$ & \\
\hline \multirow[t]{2}{*}{ Gender } & Male & 189 (67.3) & 496 (64.9) & 0.049 & $175(66.0)$ & $177(66.8)$ & 0.016 \\
\hline & Female & $92(32.7)$ & $268(35.1)$ & & $90(34.0)$ & $88(33.2)$ & \\
\hline \multirow{3}{*}{ Age category (years) } & $15-34$ & $121(43.1)$ & 354 (46.3) & 0.067 & 117 (44.2) & $126(47.5)$ & 0.068 \\
\hline & $35-50$ & $99(35.2)$ & 257 (33.6) & & $90(34.0)$ & $85(32.1)$ & \\
\hline & More than 50 & $61(21.7)$ & $153(20.0)$ & & $58(21.9)$ & $54(20.4)$ & \\
\hline Age, years (continuous) & Mean (SD) & 40.16 (15.59) & 38.20 (15.28) & 0.127 & 39.85 (15.65) & 39.18 (15.62) & 0.042 \\
\hline \multirow{2}{*}{$\begin{array}{l}\text { Type of person with bacteriologically } \\
\text { confirmed pulmonary tuberculosis }\end{array}$} & New diagnosis & $235(83.6)$ & $681(89.1)$ & 0.161 & $230(86.8)$ & $225(84.9)$ & 0.054 \\
\hline & Previously treated & $46(16.4)$ & $83(10.9)$ & & 35 (13.2) & $40(15.1)$ & \\
\hline \multirow[t]{2}{*}{ Mode of diagnosis of tuberculosis } & Microscopy & 247 (87.9) & 517 (67.7) & 0.502 & $231(87.2)$ & 225 (84.9) & 0.065 \\
\hline & GeneXpert & 34 (12.1) & 247 (32.3) & & $34(12.8)$ & $40(15.1)$ & \\
\hline \multirow[t]{3}{*}{ Antituberculosis regimen } & 2RHZE/4RH & $151(53.7)$ & $479(62.7)$ & 0.246 & $146(55.1)$ & $163(61.5)$ & 0.130 \\
\hline & 2RHZE/6HE & 98 (34.9) & $244(31.9)$ & & 96 (36.2) & $82(30.9)$ & \\
\hline & 2RHZES/1RHZE/5RHE & $32(11.4)$ & $41(5.4)$ & & $23(8.7)$ & $20(7.5)$ & \\
\hline \multirow[t]{2}{*}{ HIV infected } & No & 204 (72.6) & $528(69.1)$ & 0.077 & $192(72.5)$ & 188 (70.9) & 0.034 \\
\hline & Yes & 77 (27.4) & $236(30.9)$ & & $73(27.5)$ & $77(29.1)$ & \\
\hline \multirow{2}{*}{$\begin{array}{l}\text { Form of directly observed therapy } \\
\text { short course }\end{array}$} & Facility & $17(6.0)$ & $30(3.9)$ & 0.098 & $11(4.2)$ & $14(5.3)$ & 0.053 \\
\hline & Community & 264 (94.0) & $734(96.1)$ & & $254(95.8)$ & 251 (94.7) & \\
\hline \multirow[t]{2}{*}{ Treatment supporter availability } & No & $248(88.3)$ & $670(87.7)$ & 0.017 & $237(89.4)$ & $243(91.7)$ & 0.078 \\
\hline & Yes & $33(11.7)$ & 94 (12.3) & & 28 (10.6) & $22(8.3)$ & \\
\hline
\end{tabular}

greatest reduction in pseudo- $R^{2}$ value with over $90 \%$ drop. These two measures indicate that propensity score-matched analysis was successful.

Effect of SITT on treatment success rate. Table 4 presents treatment outcome analysis before and after propensity score-matched analysis. Before propensity score-matched analysis, 219 (69.8\%) participants in the DIT group and 545 $(71.3 \%)$ in the SITT group achieved treatment success, although the difference was not statistically significant $(P=$ $0.617)$. A binary logistic regression analysis showed that SITT was not significantly associated with treatment success rate at both unadjusted (unadjusted OR, 1.08; 95\% Cl: $0.80-1.46$ ) and adjusted (adjusted OR, 0.97; 95\% Cl: $070-1.33$ ) analyses. In the propensity score-matched cohort data, the treatment success rate in DIT and SITT groups was not any different: $184(69.4 \%)$ versus 370 (69.8\%), respectively $(P=0.850)$, and binary logistic regression analysis confirmed SITT had no statistically significant effect on treatment success rate (unadjusted OR, 0.96; 95\% Cl: $0.67-1.40)$.

Sensitivity analysis showed that when gamma is 1.7 , there was a shift from statistically nonsignificant to statistically significant odds of lower bounds. Because gamma of 1.7 is relatively distant from 1 , a point of no hidden bias, this implied that the propensity score-matched analysis results are robust to hidden bias or unobserved confounders.

\section{DISCUSSION}

Our study in rural eastern Uganda sheds light on the success of implementing SITT, the role of GeneXpert in the success of SITT, and whether SITT influences treatment success rate. Our data show that $73 \%$ of adult persons with BC-PTB in rural eastern Uganda had SITT, which is far below the desired $100 \%$ target of the WHO policy statement on same-day diagnosis and treatment. ${ }^{7}$ Most of the literature reviewed shows that many tuberculosis control programs in resource-limited settings such as Uganda have failed to reach the $100 \%$ WHO recommendation of SITT. For instance, a study conducted in Pakistan at 48 private healthcare facilities shows that $66 \%$ of persons with tuberculosis were initiated on treatment either on the same day as diagnosis or on the following day, ${ }^{28}$ which is much lower than what we observed in this study. A study in Haiti among adolescents ${ }^{29}$ indicates SITT of $80 \%$, fairly close to what we report in this study. A study in Zimbabwe reported a mean delay of 30 days from diagnosis of tuberculosis to initiation of antituberculosis treatment (treatment delay), although this significantly declined between 2012 and 


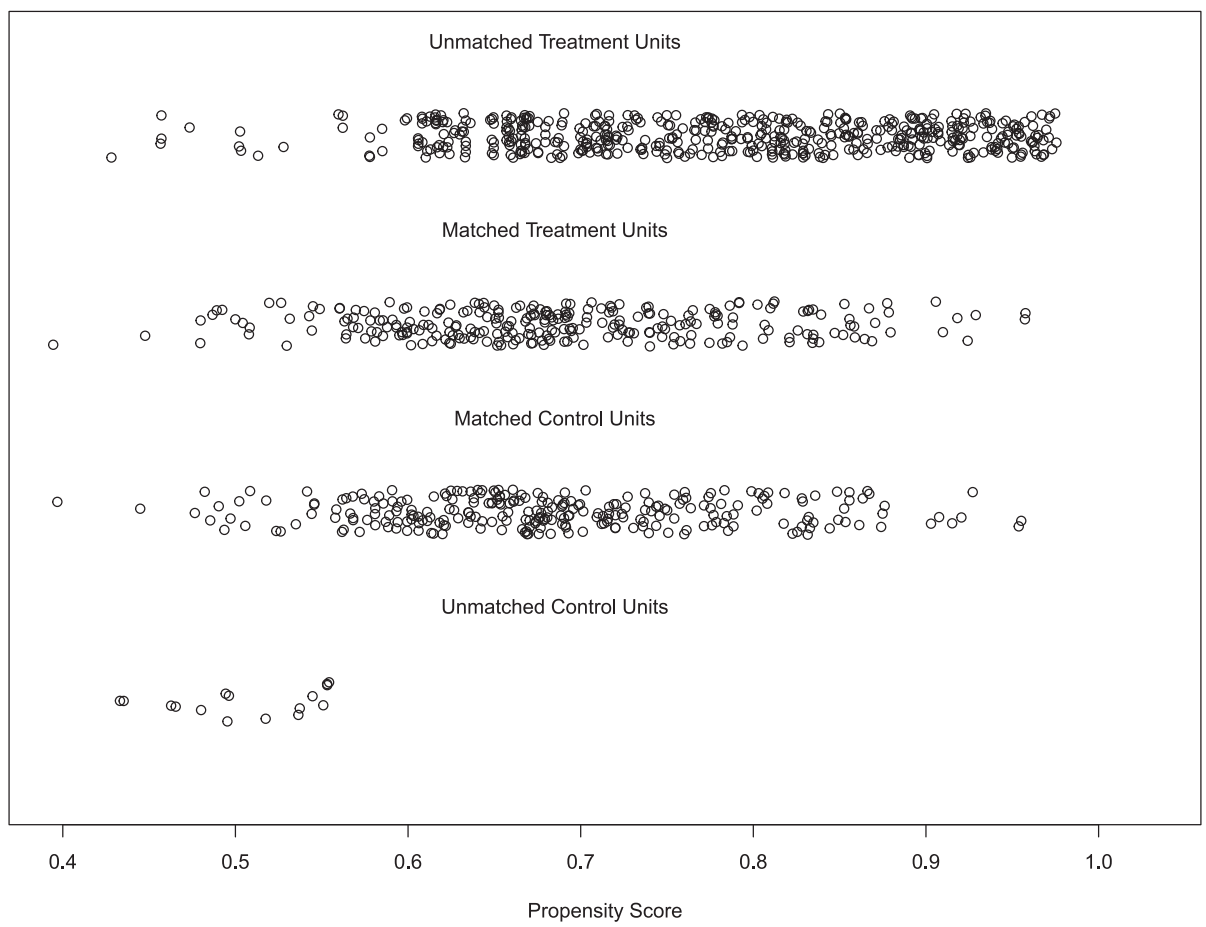

FIGURE 1. Jitter plot of propensity score distribution before and after matching. Note: 1) Unmatched treated units: unmatched same-day initiation of tuberculosis treatment (SITT) group; 2) matched treated units: matched SITT group; 3) matched control units: matched delayed initiation of treatment (DIT) group; 4) unmatched control units: unmatched DIT group.

$2016 .{ }^{30}$ All of these data confirm that many tuberculosis programs are struggling with lengthy delays between diagnosis and treatment initiation, despite the increased risk of tuberculosis transmission at household, community, and population levels posed by low SITT. Concerning DIT, we recognize that the reasons for DIT are context specific and it may not be appropriate to compare Uganda with other individual country experiences. We recommend that in future, qualitative studies should be conducted in Uganda to understand these reasons.

This study shows that persons with newly diagnosed BCPTB are more likely to receive SITT than previously treated persons with BC-PTB. Consistent with findings of an earlier study, persons previously treated for tuberculosis have increased tendency to delay treatment initiation by about 7 days compared with those with new tuberculosis diagnosis. ${ }^{31}$ The reasons for delayed treatment initiation are not clear, but we attempt to explain some.

One plausible reason might be that health workers may perceive persons with new BC-PTB diagnosis as a priority, and this probably may place them at higher likelihood of SITT. Although this explanation seems credible, there are contradicting findings elsewhere. A study in Zimbabwe shows that persons with new tuberculosis diagnosis have longer delays in treatment initiation due to lack of prior contact or experience with tuberculosis services. ${ }^{30}$ However, unlike the present study, the Zimbabwean study defined DIT as $\geq 3$ days between diagnosis of tuberculosis and treatment initiation. Also, health system factors might have played a role in influencing the observed differences, particularly for previously treated persons with BC-PTB.

Our study shows that diagnosis of tuberculosis by GeneXpert is associated with more likelihood of SITT than sputum smear microscopy. In Uganda, a recent study found that the introduction of GeneXpert improved the proportion of smearnegative persons with tuberculosis who are initiated on treatment at five rural tertiary hospitals by almost 2-fold, from $5.9 \%$ to $10.8 \% .^{32}$ On-site GeneXpert provides more rapid and accurate diagnosis of MTB in sputum specimens than microscopy. ${ }^{10}$ The fast turnaround of test results increases the

TABLE 4

Effect of SITT on treatment success rate among persons with bacteriologically confirmed pulmonary tuberculosis in rural eastern Uganda

\begin{tabular}{|c|c|c|c|c|c|c|c|c|c|c|}
\hline \multirow{2}{*}{ 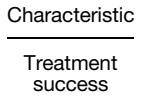 } & \multicolumn{6}{|c|}{ Before propensity score-matched analysis } & \multicolumn{4}{|c|}{ After propensity score-matched analysis } \\
\hline & $\operatorname{DIT}(n=281)$ & $\operatorname{SITT}(n=764)$ & & $\begin{array}{l}\text { sted analysis } \\
95 \% \mathrm{Cl})\end{array}$ & & $\begin{array}{l}\text { ted analysis } \\
\text { \{, } 95 \% \mathrm{Cl} \text { ) }\end{array}$ & $\operatorname{DIT}(n=265)$ & $\operatorname{SITT}(n=265)$ & & $\begin{array}{l}\text { ne analysis } \\
95 \% \mathrm{Cl})\end{array}$ \\
\hline No & 85 (30.2) & $196(28.7)$ & 1 & - & 1 & - & 79 (29.8) & $81(30.6)$ & 1 & - \\
\hline Yes & $219(69.8)$ & 545 (71.3) & 1.08 & $(0.80-1.46)$ & 0.97 & $(0.70-1.33)$ & 186 (70.2) & $184(69.4)$ & 0.96 & $(0.67-1.40)$ \\
\hline
\end{tabular}

AOR = adjusted odds ratio; $\mathrm{DIT}$ = delayed initiation of tuberculosis treatment; $\mathrm{OR}$ = odds ratio; SITT = same-day initiation of tuberculosis treatment. Odds ratios are exponentiated coefficients; $95 \% \mathrm{Cl}$ for $\mathrm{OR}$ in brackets.

${ }^{*} P<0.05$.

** $P<0.01$.

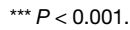


chance of SITT. However, our study shows no difference in SITT between sites with and without GeneXpert, and this could be due to one of two reasons. The first is that the hub system for pickup and testing of samples is very efficient and matches the pace at health facilities with on-site GeneXpert. The second explanation is the possibility that the opposite is true.

It is important to recognize that sputum smear microscopy has several challenges. For example, sputum smear microscopy is labor intensive and, given the large volume of samples to be processed, requires more human resources than is available. Therefore, there is likelihood that some samples might not be examined on the same day as consultation or collection, leading to diagnostic and treatment delays. The rollout of GeneXpert, a faster diagnostic tool that generates results within a few hours, ${ }^{33}$ addresses the limitations of sputum smear microscopy.

Our finding supports the strengthening of GeneXpert expansion and integration in laboratories at peripheral sites to reduce diagnostic turnaround time lags and accelerate clinical decisions.

Our study shows that SITT has no effect on treatment success rate among adult persons with BC-PTB in this rural population. This result should be interpreted cautiously. Our analysis was based on the WHO policy guide for same-day diagnosis as consultation and consequently SITT. We considered treatment initiation after the day of diagnosis as DIT. Moreover, the median time to treatment in the DIT is 2 days, with an interquartile range of 1-10 days, which may arguably have little impact on overall clinical outcomes. We also recognize that tuberculosis treatment is accompanied by numerous challenges at personal and community levels that all influence treatment success rate. These factors include adverse side effects of antituberculosis drugs, suboptimal adherence to antituberculosis drugs, inadequate treatment support, stigma, and interaction between tuberculosis and other comorbidities, for instance, diabetes mellitus, which were not included in the analysis.

Overall, SITT has important benefits in reducing tuberculosis transmission and achieving good treatment outcomes as demonstrated in different studies. One study reports that delays in treatment initiation exceeding 30 days from the onset of tuberculosis symptoms to treatment initiation increase the risk of unsuccessful treatment outcome by almost 2-fold. ${ }^{34}$ Data from Brazil show that the replacement of sputum smear microscopy with GeneXpert to enable SITT resulted in increased bacteriological confirmation of pulmonary tuberculosis and accurate detection of rifampicin resistance. ${ }^{35}$ Another study shows that SITT reduces patient costs associated with frequent travels to health facilities ${ }^{33}$ and tuberculosis transmission at household and community levels. ${ }^{4}$ One large multicountry study shows that the use of GeneXpert (with potential to enhance SITT) is associated with lower likelihood of unsuccessful treatment outcome. ${ }^{36}$

Study strengths and limitations. Our study has some strengths. First, it is among the first studies to assess the effect of implementing the WHO policy statement on same-day diagnosis and initiation of tuberculosis on treatment success rate in rural sub-Saharan Africa. Second, we used robust methods to determine the unbiased effect of SITT on treatment outcomes using propensity score-matched analysis, which approximates a randomized control trial by creating comparable groups with respect to confounders and removing selection bias. Third, the sample size for both the primary and secondary analyses was sufficiently large.
However, there are some limitations to consider. First, the study findings do not apply to persons with tuberculosis younger than 15 years and other forms of tuberculosis, namely, clinically diagnosed and extrapulmonary tuberculosis. Second, we did not have data on severity of tuberculosis disease and its symptoms as a covariate in primary analysis, yet it potentially influences diagnostic and treatment decisions and outcomes. Third, the propensity score-matched analysis included only measured covariates, and several unmeasured covariates such as adherence to antituberculosis drugs and complex interaction between tuberculosis and other comorbidities were not included in the analysis. However, sensitivity analysis showed that the results of the propensity score-matched analysis are robust to unmeasured confounders and the matching approach.

\section{CONCLUSION AND RECOMMENDATIONS}

Our study shows that implementation of SITT among adult persons with BC-PTB in rural eastern Uganda is suboptimal and may, therefore, pose potential public health challenges of tuberculosis transmission at household and community levels. Our study also shows that GeneXpert increases the likelihood of SITT, but SITT has no effect on treatment success rate. We recommend that local and national tuberculosis control programs should support the implementation of the WHO policy statement on same-day diagnosis of tuberculosis and treatment to enhance SITT. An operational research is needed to understand the mechanisms for successful implementation of GeneXpert and SITT in rural areas.

Received December 5, 2019. Accepted for publication July 8, 2020.

Published online August 10, 2020.

Acknowledgments: We are indebted to the German Academic Exchange Services (DAAD) for awarding the primary author a scholarship to pursue doctoral studies at Mbarara University of Science and Technology, Uganda. We also acknowledge the district health officers of Soroti, Kumi, Serere, and Ngora districts for granting administrative clearance to collect tuberculosis data at respective study sites. We appreciate the support given by the district tuberculosis and leprosy supervisors of Serere, Soroti, Kumi, and Ngora districts; the tuberculosis focal persons of respective study sites; and the research assistants. We extend our gratitude to the East Africa Social Science Translation (EASST) Collaborative and the Center for Effective Global Action (CEGA) at the University of California, Berkley, for organizing the training on impact evaluation that supported the design and analysis of this manuscript. In particular, we thank Damazo T. Kadengye. The American Society of Tropical Medicine and Hygiene (ASTMH) assisted with publication expenses.

Authors' addresses: Jonathan Izudi, Imelda K. Tamwesigire, and Francis Bajunirwe, Department of Community Health, Faculty of Medicine, Mbarara University of Science and Technology, Mbarara, Uganda, E-mails: jonahzd@gmail.com, itamwesigire@gmail.com, and fbaj@yahoo.com.

\section{REFERENCES}

1. World Health Organization, 2019. Global Tuberculosis Report 2019. Geneva, Switzerland: WHO.

2. World Health Organization, 2020. Tuberculosis: Key Facts. Available at: https://www.who.int/news-room/fact-sheets/detail/tuberculosis. Accessed May 12, 2020.

3. Adenager GS, Alemseged F, Asefa H, Gebremedhin AT, 2017. Factors associated with treatment delay among pulmonary tuberculosis patients in public and private health facilities in Addis Ababa, Ethiopia. Tuberc Res Treat 2017: 5120841. 
4. World Health Organization, 2017. Guidelines for Treatment of Drug-Susceptible Tuberculosis and Patient Care, 2017 Update. Geneva, Switzerland: WHO.

5. Shiferaw MB, Zegeye AM, 2019. Delay in tuberculosis diagnosis and treatment in Amhara state, Ethiopia. BMC Health Serv Res 19: 232.

6. Virenfeldt J, Rudolf F, Camara C, Furtado A, Gomes V, Aaby P, Petersen E, Wejse C, 2014. Treatment delay affects clinical severity of tuberculosis: a longitudinal cohort study. BMJ Open 4: e004818.

7. World Health Organization, 2011. Same-Day Diagnosis of Tuberculosis by Microscopy: Policy Statment. Geneva, Switzerland: WHO.

8. Lopes A et al., 2019. Prospective study comparing the conventional and same-day strategies to diagnose pulmonary tuberculosis. Med Mal Infect 50: 36-42.

9. Davis JL, Cattamanchi A, Cuevas LE, Hopewell PC, Steingart KR, 2013. Diagnostic accuracy of same-day microscopy versus standard microscopy for pulmonary tuberculosis: a systematic review and meta-analysis. Lancet Infect Dis 13: 147-154.

10. Tang T, Liu F, LuX, Huang Q, 2017. Evaluation of GeneXpert MTB/ RIF for detecting Mycobacterium tuberculosis in a hospital in China. J Int Med Res 45: 816-822.

11. Republic of Uganda, 2018, Ministry of Health Annual Health Sector Perofrmance Report 2016/2017. Kampala, Uganda: Ministry of Health.

12. Republic of Uganda, 2019. Annual Health Sector Peformance Report 2017/2018. Kampala, Uganda: Ministry of Health.

13. Republic of Uganda, 2016. The Uganda National Tuberculosis Prevalence Survey: 2014-2015 Survey Report. Kampala: Ministry of Health.

14. World Health Organization, 2003. Treatment of Tuberculosis: Guidelines for National Programmes. Geneva, Switzerland: WHO.

15. Starks H, Garrido MM, 2004. Observational \& Quasi-Experimental Research Methods. 8th Annual Kathleen Foley Palliative Care Retreat Method Workshop Google Scholar, 2004.

16. Smith JA, Todd PE, 2005. Does matching overcome LaLonde's critique of nonexperimental estimators? J Econometrics 125: 305-353.

17. Schmidt OC, Kohlmann T, 2008. When to use the odds ratio or the relative risk? Int J Public Health 53: 165-167.

18. Okoli G, Sanders R, Myles P, 2014. Demystifying propensity scores. Br J Anaesth 112: 13-15.

19. Thavaneswaran A. Propensity Score Matching in Observational Studies. Manitoba Center for Health Policy. Winnipeg, Canada: University of Manitoba. Available at: https://www.umanitoba. ca/faculties/health_sciences/medicine/units/chs/departmental_ units/mchp/protocol/media/propensity_score_matching.pdf. Accessed November 11, 2019.

20. Olmos A, Govindasamy P, 2015. Propensity scores: a practical introduction using R. J Multidiscip Eval 11: 68-88.

21. R Core Team, 2018. R: A Language and Environment for Statistical Computing. R Foundation for Statistical Computing. Available at: https://www.R-project.org/.2019. Accessed June 5, 2019.
22. Ho D, Imai K, King G, Stuart E, 2007. Matching as nonparametric preprocessing for reducing model dependence in parametric causal inference. Polit Anal 15: 199-236.

23. Ho D, Imai K, King G, Stuart E, 2007. Matchit: nonparametric preprocessing for parametric causal inference. J Stat Softw 42: 9-16.

24. Vandenbroucke JP, Von Elm E, Altman DG, Gøtzsche PC, Mulrow CD, Pocock SJ, Poole C, Schlesselman JJ, Egger M, STROBE Initiative, 2007. Strengthening the reporting of observational studies in epidemiology (STROBE): explanation and elaboration. PLoS Med 4: e297.

25. Von Elm E, Altman DG, Egger M, Pocock SJ, Gøtzsche PC, Vandenbroucke JP, STROBE Initiative, 2014. The strengthening the reporting of observational studies in epidemiology (STROBE) statement: guidelines for reporting observational studies. Int J Surg 12: 1495-1499.

26. Yao XI, Wang X, Speicher PJ, Hwang ES, Cheng P, Harpole DH, Berry MF, Schrag D, Pang HH, 2017. Reporting and guidelines in propensity score analysis: a systematic review of cancer and cancer surgical studies. J Natl Cancer Inst 109: djw323.

27. Staffa SJ, Zurakowski D, 2018. Five steps to successfully implement and evaluate propensity score matching in clinical research studies. Anesth Analg 127: 1066-1073.

28. Ali SM et al., 2018. Loss-to-follow-up and delay to treatment initiation in Pakistan's national tuberculosis control programme. BMC Public Health 18: 335.

29. Reif $L$ et al., 2018. Outcomes across the tuberculosis care continuum among adolescents in Haiti. Public Health Action 8: 103-109.

30. Mugauria H, Shewadeb DH, Dlodlob AR, Hoved S, Sibanda E, 2018. Bacteriologically confirmed pulmonary tuberculosis patients: loss to follow-up, death and delay before treatment initiation in Bulawayo, Zimbabwe from 2012-2016. Int J Infect Dis 76: 6-13.

31. Getnet F, Demissie M, Assefa N, Mengistie B, Worku A, 2017. Delay in diagnosis of pulmonary tuberculosis in low-and middle-income settings: systematic review and metaanalysis. BMC Pulm Med 17: 202.

32. Zawedde-Muyanja S, Manabe YC, Sewankambo N, Nakiyingi L, Nakanjako D, 2018. Xpert ${ }^{\circledR}$ MTB/RIF associated with improved treatment initiation among patients with smear-negative tuberculosis. Int J Tuberc Lung Dis 22: 1475-1480.

33. Kirwan ED, Gilman HR, 2012. Same-day diagnosis and treatment of tuberculosis. Lancet 13: 102-104.

34. Asres A, Jerene D, Deressa W, 2018. Delays to treatment initiation is associated with tuberculosis treatment outcomes among patients on directly observed treatment short course in Southwest Ethiopia: a follow-up study. BMC Pulm Med 18: 64.

35. Durovni B, Saraceni V, van den Hof S, Trajman A, Cordeiro-Santos M, Cavalcante S, Menezes A, Cobelens F, 2014. Impact of replacing smear microscopy with Xpert MTB/RIF for diagnosing tuberculosis in Brazil: a stepped-wedge clusterrandomized trial. PLoS Med 11: e1001766.

36. Clouse $\mathrm{K}$ et al., 2017. Low implementation of Xpert MTB/RIF among HIV/TB co-infected adults in the International epidemiologic Databases to Evaluate AIDS (leDEA) program. PLoS One 12: e0171384. 